\title{
Treatment of Methicillin-Resistant Staphylococcus aureus (MRSA) Pneumonia with Ceftaroline Fosamil in a Patient with Inhalational Thermal Injury
}

\author{
Janie Faris · Ryan P. Mynatt $\cdot$ Ashley D. Hall Snyder $\cdot$ Michael J. Rybak
}

To view enhanced content go to www.infectiousdiseases-open.com

Received: August 14, 2015 / Published online: November 5, 2015

(C) The Author(s) 2015. This article is published with open access at Springerlink.com

\section{ABSTRACT}

A 48-year-old female, who was found unresponsive and suffered inhalation injury secondary to a house fire, was transferred to our burn center for definitive treatment. Post tracheostomy, the patient became febrile and tachycardic. On hospital day (HD) 5, the patient expressed thick yellow secretions during suctioning and diffuse rhonchi was noted on

Electronic supplementary material The online version of this article (doi:10.1007/s40121-015-0096-4) contains supplementary material, which is available to authorized users.

J. Faris

Trauma Surgery Critical Care, Department of Pharmacy, Carilion Roanoke Memorial Hospital, 1906 Belleview Avenue, Roanoke, VA 24014, USA

R. P. Mynatt

Detroit Receiving Hospital, Wayne State University,

Detroit, MI, USA

\section{A. D. Hall Snyder · M. J. Rybak}

Anti-Infective Research Laboratory, Eugene Applebaum College of Pharmacy and Health

Sciences, Wayne State University, Detroit, MI, USA

M. J. Rybak ( $ه)$

School of Medicine, Wayne State University,

Detroit, MI, USA

e-mail:m.rybak@wayne.edu physical exam. Blood cultures and a culture from the broncheo-alvelolar lavage grew Gram-positive cocci in clusters and the patient was started on empiric vancomycin. Despite aggressive vancomycin dosing (1750 mg intravenously every $6 \mathrm{~h}$ ), the patient's status continued to deteriorate. The organism was identified as methicillin-resistant Staphylococcus aureus (MRSA) with a vancomycin minimum inhibitory concentration (MIC) of $2 \mathrm{mg} / \mathrm{L}$. Based on the potential for drug-drug interactions with linezolid, the patient was started on ceftaroline fosamil (MIC $=0.5 \mathrm{mg}$ / L) $600 \mathrm{mg}$ intravenously every $8 \mathrm{~h}$ with a prolonged 2-h infusion to anticipate suboptimal concentrations secondary to thermal burn injury. Post change in antibiotic therapy, a rapid clinical improvement was observed with the patient becoming afebrile at $48 \mathrm{~h}$ after initiation of ceftaroline. The patient completed a total of 14 days of ceftaroline therapy and was subsequently weaned from the ventilator on HD 22 and decannulated 2 days later. To our knowledge, this is the first report of the use of ceftaroline for the treatment of MRSA pneumonia in a patient with thermal injury. 
Keywords: Burn; Ceftaroline; Inhalational; Pharmacokinetics; Pneumonia; Methicillin-resistant Staphylococcus aureus (MRSA); Staphylococci

\section{INTRODUCTION}

Infections caused by methicillin-resistant Staphylococcus aureus (MRSA) are a frequent occurrence in most medical centers, especially in patients admitted to the intensive care unit (ICU) for prolonged periods of time. These infections can be secondary to implanted catheters, skin and soft tissue infections, and pneumonia. Patients with extensive thermal injuries are at high risk of developing infections from MRSA and other multi-drug-resistant pathogens due to their need for prolonged ICU care, frequent receipt of antimicrobial therapy, and the immunological and physiological disturbances encountered as a result of their injuries. Historically vancomycin, a glycopeptide antimicrobial, has been the treatment of choice for MRSA infections. However, the clinical utility of vancomycin has recently been questioned when employed in the treatment of MRSA infections when the mean inhibitory concentration (MIC) of vancomycin is greater than $1 \mathrm{mg} / \mathrm{L}$, with published literature reporting increased treatment failure and mortality in patients due to inability to attain currently established pharmacokinetic (PK) and pharmacodynamic (PD) targets [1-3].

Ceftaroline fosamil (Teflaro $^{\circledR}$; Actavis Pharmaceuticals), an advanced cephalosporin with activity against MRSA, was approved by the Food and Drug Administration (FDA) for the treatment of acute bacterial skin and skin structure infections and community-acquired bacterial pneumonia (CABP). We describe a case of MRSA pneumonia in a patient with inhalational thermal injuries who did not respond to initial vancomycin therapy (vancomycin $\mathrm{MIC}=2 \mathrm{mg} / \mathrm{L}$ ) but responded clinically to treatment with ceftaroline. Currently, there are limited data available to support the utilization of ceftaroline for the treatment of pneumonia caused by MRSA. There are no data that we are aware of with respect to treatment of patients with burn-related infections. This article does not contain any new studies with human or animal subjects performed by any of the authors.

\section{CASE REPORT}

A 48-year-old Caucasian female was found unresponsive at the scene of a house fire with burns to the face $[<1 \%$ total body surface area (BSA)] and inhalation injury. She was intubated via endotracheal tube at an outside facility and subsequently transferred to our burn center for definitive care. The initial bronchoscopy showed diffuse carbonaceous sputum with pale friable mucosa extending throughout the airways distally. The patient was intubated for airway protection, which required adequate sedation and neuromuscular blockade. Due to the inability of the patient to provide a detailed medical history, the patient's sister and psychiatrist supplied the relevant information, which included a diagnosis of severe depression with psychotic features. On admission, the patient's list of home medications included paliperidone palmitate $234 \mathrm{mg}$ once monthly as an intramuscular injection, paliperidone $6 \mathrm{mg}$ by mouth daily, citalopram $40 \mathrm{mg}$ by mouth daily, trazodone $150 \mathrm{mg}$ by mouth at bedtime, risperidone $2 \mathrm{mg}$ by mouth twice daily, and clonazepam $1 \mathrm{mg}$ by mouth twice 
daily. The patient had no known drug allergies or other relevant medical history. She was a chronic smoker and substance abuser, but on admission the urine drug screen was negative.

Upon admission to the burn ICU, the patient had a mild leukocytosis (white blood cell count $15.8 \mathrm{k} / \mathrm{mm}^{3}$ ) but was afebrile, normotensive, and demonstrated adequate urine output $(>0.5 \mathrm{~mL} /$ $\mathrm{kg} / \mathrm{hr}$ ). She had evidence of acute respiratory distress syndrome (ARDS), with a ratio of partial pressure arterial oxygen and fraction of inspired oxygen of less than 200, and was started on pressure control ventilation. Due to the severity of the inhalation injury and the need for prolonged mechanical ventilation with aggressive pulmonary hygiene, a tracheostomy was performed on the second hospital day (HD). Post-operatively, the patient became febrile with an associated tachycardia. By HD 5, the patient expressed thick yellow secretions during suctioning and on physical examination diffuse rhonchi were appreciated, worse in the right lung than the left, corresponding with a worsening right-sided, patchy infiltrate seen on chest X-ray. A culture from the broncheo-alvelolar lavage (BAL) on HD 6 revealed gram-positive cocci in clusters (Table 1). Blood cultures obtained on HD 4 also grew Gram-positive cocci in clusters, and the patient was started on empiric vancomycin and cefepime on HD 6 for presumed

Table 1 Cultures and susceptibilities of select antibiotics

\begin{tabular}{|c|c|c|c|}
\hline Date & Site & Organism & Susceptibility (mg/L) \\
\hline \multirow[t]{4}{*}{ HD 4} & Blood & MRSA & Daptomycin: $\mathrm{MIC}<0.5$ \\
\hline & & & Gentamicin: MIC $<4$ \\
\hline & & & TMP/SMX: $\mathrm{MIC}<0.5 / 9.5$ \\
\hline & & & Vancomycin: $\mathrm{MIC}=1$ \\
\hline \multirow[t]{4}{*}{ HD 4} & Respiratory & MRSA & Gentamicin: $\mathrm{MIC}<0.5$ \\
\hline & & & Linezolid: MIC < 4 \\
\hline & & & TMP/SMX: $\mathrm{MIC}<0.5 / 9.5$ \\
\hline & & & Vancomycin: $\mathrm{MIC}=1$ \\
\hline \multirow[t]{5}{*}{ HD 6} & Quantitative BAL & $>180,000 \mathrm{CFU} / \mathrm{mL}$ MRSA & Gentamicin: $\mathrm{MIC}<4$ \\
\hline & & & Linezolid: MIC $<4$ \\
\hline & & & TMP/SMX: MIC < 0.5/9.5 \\
\hline & & & Vancomycin: $\mathrm{MIC}=2$ \\
\hline & & & Ceftaroline: $\mathrm{MIC}=0.5$ \\
\hline \multirow[t]{4}{*}{ HD 10} & Quantitative BAL & $>110,000 \mathrm{CFU} / \mathrm{mL}$ MRSA & Gentamicin: MIC $<4$ \\
\hline & & & Linezolid: MIC $<4$ \\
\hline & & & TMP/SMX: $\mathrm{MIC}<0.5 / 9.5$ \\
\hline & & & Vancomycin: $\mathrm{MIC}=2$ \\
\hline HD 10 & Blood & No growth & \\
\hline
\end{tabular}

$B A L$ broncheo-alvelolar lavage, $C F U$ colony forming units, $H D$ hospital day, $M I C$ mean inhibitory concentration, $M R S A$ methicillin-resistant Staphylococcus aureus, TMP/SMX trimethoprim/sulfamethoxazole 
hospital-acquired pneumonia with associated bacteremia. However, despite an aggressive vancomycin dosing schedule [1750 mg intravenous (IV) every $6 \mathrm{~h}$ ] and adequate, supratherapeutic, vancomycin trough concentrations of $26 \mathrm{mg} / \mathrm{L}$, the patient's clinical status did not improve. She continued to be febrile and tachycardic, requiring increased ventilatory support and oxygenation requirements. Repeat urine and blood cultures obtained on HD 5 were negative for the Gram-positive pathogen, which was at this time identified as MRSA. The repeat bronchoscopy on HD 10 demonstrated mild improvement in secretions, but the Gram-stain from the BAL subsequently yielded growth in culture of $>110,000$ colony forming units $/ \mathrm{mL}$ gram-positive cocci in clusters, later identified as MRSA with a vancomycin MIC of $2 \mathrm{mg} / \mathrm{L}$ by automated susceptibility testing. Due to the patient's deteriorating clinical status on HD 10 while on high-dose vancomycin therapy, the decision was made to modify the antibiotic regimen. At the Detroit Medical Center, in accordance with suggested treatment pathways, MRSA bacteremia and/or pneumonia and a vancomycin $\mathrm{MIC}$ of $\geq 2 \mathrm{mg} / \mathrm{L}$ constitute a reason to change therapy to a suitable alternative. However, with consideration of the patient's medication history, and current medication regimen of antipsychotics and a selective serotonin reuptake inhibitor (SSRI), linezolid was not employed due to the possibility of drug-drug interactions with the psychiatric medications. After consultation with the infectious diseases (ID) pharmacist and the ID medical consult service, a decision was made to initiate ceftaroline fosamil (Teflaro) for the treatment of this patient's MRSA pneumonia.

Ceftaroline fosamil (Teflaro) is a new advanced generation cephalosporin approved by the FDA for use in acute bacterial skin and skin structure infection (ABSSSI) including MRSA and CABP caused by susceptible bacteria. Ceftaroline binds to the penicillin-binding proteins including PBP 2a associated with methicillin resistance in $S$. aureus and prevents the cross-linkage of peptidoglycan in bacterial cell walls [4-6]. Ceftaroline fosamil, a prodrug, is converted to the active form of ceftaroline via serum phosphatases and undergoes minimal cytochrome $\mathrm{P} 450$ oxidation. Approximately $88 \%$ of a dose of ceftaroline is excreted renally, with only $6 \%$ of the drug recovered in the feces [4]. The prescribing information indicates the approved dose of ceftaroline fosamil administered intravenously is $600 \mathrm{mg}$ every $12 \mathrm{~h}$ for ABSSSI and CABP [7]. However, due to the increased volume of distribution $\left(V_{\mathrm{d}}\right)$, renal clearance, and urine output in burn injury patients, the patient's regimen was empirically modified to $600 \mathrm{mg}$ IV every $8 \mathrm{~h}$, and administered as a 2 -h infusion to ensure that adequate drug concentrations be maintained throughout the dosing interval. The clinical MRSA isolate tested had a ceftaroline MIC of $0.5 \mathrm{mg} / \mathrm{L}$ by Etest $^{\circledR}$ (bioMérieux SA). This antimicrobial regimen was continued for a total of 14 days. The patient became afebrile $48 \mathrm{~h}$ after initiation of ceftaroline and remained afebrile for the length of the hospital stay. Rapid clinical improvement was seen after initiation of ceftaroline; the patient was subsequently weaned from the ventilator on HD 22 and decannulated 2 days later. However, because of her psychiatric illness and auditory/visual hallucinations, hospital discharge was delayed to allow for psychiatric placement.

Due to the relative lack of clinical data supporting the use of ceftaroline in burn patients, serum concentrations of ceftaroline were obtained for PK characterization and 
Table 2 Patient-specific ceftaroline levels

\begin{tabular}{ll}
\hline Time & Ceftaroline level $(\mathbf{m g} / \mathrm{L})$ \\
\hline $0615-0815$ & Infusion $600 \mathrm{mg}$ IV ceftaroline \\
0845 & $21.9^{\mathrm{a}}$ \\
1045 & 7.3 \\
1330 & 4.2 \\
\hline
\end{tabular}

$I V$ intravenous

a 30 min post-infusion

potential dosage adjustment. Serum drug levels were obtained on day five of ceftaroline therapy. Concentrations were obtained $30 \mathrm{~min}$ post-infusion, $2 \mathrm{~h}$ later, and a final concentration drawn 30 min prior to the next dose (Table 2).

\section{Serum Drug Assay and PK Characterization}

The ceftaroline bioassay was performed according to previously published methods [8]. Briefly, quarter-inch disks were placed on agar plates (antibiotic medium number 11) pre-swabbed with Bacillus subtilis ATCC 6633. The disks were spotted with $10 \mu \mathrm{L}$ of ceftaroline standards $(2.5,10$, and $40 \mathrm{mg} / \mathrm{L})$ or plasma samples. Each standard and sample was tested in duplicate. Plates were incubated for $18-24 \mathrm{~h}$ at $37^{\circ} \mathrm{C}$, after which the zone sizes were measured using a protocol reader (Protocol;
Microbiology International, Frederick, MD, USA). Ceftaroline half-life, $C_{\max }$ (peak), and $C_{\text {min }}$ (trough) $\mathrm{mg} / \mathrm{L}$ were determined from concentration-versus-time plots assuming a one-compartment model (Table 2). Patient-specific concentrations and time above MIC $(T>$ MIC) were calculated utilizing first-order elimination concepts (Table 3). The elimination half-life, peak and trough concentrations, and area under the curve (AUC) were calculated using PK Analyst Software (version 1.10; MicroMath Scientific Software, Salt Lake City, UT, USA).

\section{DISCUSSION}

MRSA is a frequently encountered pathogen in burn units and poses a serious health risk. As aforementioned, this risk is not limited to burn site infections but can be associated with bacteremia and pneumonia. Due to the severity of burn injuries, patients may require multiple surgical procedures, numerous invasive central catheters, and prolonged periods of mechanical ventilation. These risk factors, in addition to the decreased immunological response, can dramatically increase the mortality rates in burn patients. Treatment of these infections in this patient population can present a PK challenge for clinicians, and patients frequently require high

Table 3 Patient-specific ceftaroline PK parameters versus package insert

\begin{tabular}{lllllllc}
\hline & $\begin{array}{l}C_{\max } \\
(\mathbf{m g} / \mathbf{L})\end{array}$ & $\begin{array}{l}C_{\min } \\
(\mathbf{m g} / \mathbf{L})\end{array}$ & $T_{1 / 2}(\mathbf{h})$ & $V_{\mathbf{d}}(\mathbf{L} / \mathbf{k g})$ & $\begin{array}{l}\mathbf{A U C}_{\mathbf{0}-\tau} \\
(\boldsymbol{\mu g ~ h / m L})\end{array}$ & $\begin{array}{l}\boldsymbol{T}>\mathbf{M I C} \\
(\mathbf{h})\end{array}$ & $\begin{array}{l}\text { Clearance } \\
(\mathbf{L} / \mathbf{h})\end{array}$ \\
\hline Burn patient & 27.5 & 1.69 & 1.5 & 0.42 & 87.6 & 8 & 10 \\
Package insert & 21.3 & & $2.3-2.9$ & $0.31-0.45$ & 56.3 & & 9.6 \\
\hline
\end{tabular}

It should be noted that the $\mathrm{AUC}_{0-\tau}$ for the burn patient is for $0-8$ and $0-12 \mathrm{~h}$ per the package insert (i.e., the dosing interval)

$A U C$ area under the curve, $C_{\max }$ maximum serum concentration, $C_{\min }$ minimum serum concentration, $M I C$ mean inhibitory concentration, $T_{1 / 2}$ half-life, $V_{d}$ volume of distribution 
doses of antimicrobials to maintain adequate serum concentrations [9-14].

Patients that suffer from a thermal injury have numerous pathophysiological changes, which can dramatically alter the PK/PD of prescribed medications. The physiological changes vary based on the time elapsed from the initial burn injury. The acute phase occurs within the first $72 \mathrm{~h}$ of the burn injury and results in decreased cardiac and urine output, and increased systemic vascular resistance, which can affect the distribution and elimination of medications [15]. These alterations in cardiac output, renal clearance, and protein binding can significantly alter the PK properties of most medications including absorption, distribution, and elimination [15, 16]. The hypermetabolic phase occurs around $72 \mathrm{~h}$ after the burn injury. During this second phase of burn injury, patients will have increased levels of catecholamines, prostaglandins, glucagon, and cortisol which result in increased cardiac output and glomerular filtration rates [15]. Decreased albumin and increased acute-phase proteins resulting from the acute injury can also potentially alter the effectiveness of highly protein bound drug molecules since only free drug is available to elicit an effect $[15,16]$. Additionally, the $V_{\mathrm{d}}$ in significantly burned patients ( $>20 \%$ total BSA), or patients with an inhalation injury, can be altered due to extensive fluid loss from the burned tissue [16]. Finally, hepatic metabolism of medications varies after a burn injury $[12,13$, $15,16]$. In the hypermetabolic phase, a decrease in phase 1 metabolism can increase the half-life of medications that are hepatically metabolized and cleared, which ultimately increases the risk of toxicity to the patient. However, phase 2 metabolism, or glucuronidation, does not appear to be affected during the hypermetabolic phase of burn injury [17].

Alterations in the physiological response to a burn make dosing medications, especially antibiotics, challenging in burn patients. Although not typically discussed in burn literature, an inhalation injury elicits the same physiological response as any other thermal injury [18]. Lovering and colleagues [19] evaluated the dosing regimens of linezolid in patients with $>20 \%$ total BSA. In their study, the burn patients had increased non-renal clearance of the drug and elimination rate constants. However, the $C_{\max }$ concentrations and the $V_{\mathrm{d}}$ of linezolid were similar between the burn patients and healthy volunteers. A case report of a 27 -year-old male with a $52 \%$ total BSA burn demonstrated that the typical dose of linezolid $600 \mathrm{mg}$ IV every $12 \mathrm{~h}$ and meropenem $1 \mathrm{~g}$ IV every $8 \mathrm{~h}$ produced subtherapeutic serum concentrations in the patient, necessitating an increase in the frequency of dosing to obtain adequate concentrations of the antibiotics in serum, including $T>$ MIC [20]. Daptomycin $C_{\max }$ concentrations and AUC values have also been reported to be less (44\% and $47 \%$, respectively) in patients with thermal injury, with these patients experiencing a 77\% increase in mean clearance values compared to healthy volunteers [12]. This study found that daptomycin doses would need to be increased to $10-12 \mathrm{mg} / \mathrm{kg} / \mathrm{dose}$ to approximate drug exposures in healthy volunteers (dosed at $6 \mathrm{mg} / \mathrm{kg} / \mathrm{dose}$ ). Overall, the alterations in PK parameters in these antibiotics are variable based on burn size and renal function [16, 21]. In one study evaluating cefepime, the renal clearance and $V_{\mathrm{d}}$ were reportedly $10-30 \%$ higher in burn patients than non-burn patients [9]. As a result of available data in burn patients, the practice of increasing the 
dose, as well as the frequency of administration, may be reasonable to maintain therapeutic serum concentrations of antibiotics $[9,20,21]$.

In this case, alternative antimicrobial therapy was sought due to the patient's lack of clinical response to vancomycin therapy coupled with an elevated vancomycin MIC ( $2 \mathrm{mg} / \mathrm{L})$. As previously mentioned, although literature supports linezolid in the treatment of nosocomial pneumonia, it was not chosen due to the potential for subtherapeutic concentrations resulting from pathophysiological changes. In addition, due to the patient's medication history, there was a potential for drug-drug interactions with the patient's prescribed antipsychotic medication, which included an SSRI and linezolid. Case reports have cautioned against the use of this combination as near-fatal serotonin syndrome has been reported with linezolid's inhibition of MAO-A and B [22]. Therefore, ceftaroline was initiated using the $600 \mathrm{mg}$ dose at an 8-h dosing schedule, administered as a 2-h infusion, to maximize the PK/PD of the cephalosporin class (i.e., time-dependent killing) and allowing for infusion of other IV medications simultaneously.

This dosing regimen has been studied in in vitro PK/PD models and shown to be highly effective against MRSA, while the 12-h regimen has been studied in complicated skin and skin structure infections and for treatment of CABP [23-27]. Bhavnani et al. [28] used Monte Carlo Simulation (MCS) to simulate 2000 patient exposures with the $600 \mathrm{mg}$ IV every $12 \mathrm{~h}$ dose, evaluating the ability of this dosing regimen to attain a free drug time above the MIC ( $\%$ \% $>$ MIC) target up to $51 \%$ of the dosing interval against $S$. aureus isolates, utilizing a wide range of MICs $(0.125-16 \mathrm{mg} / \mathrm{L})$. Interestingly, against simulated isolates with MICs typically seen at this institution based upon internal data (MIC $901 \mathrm{mg} / \mathrm{L}$ ), it was reported that $600 \mathrm{mg}$ IV every $12 \mathrm{~h}$ would only achieve this PD endpoint in $76.2 \%$ of the simulated patients with normal renal function [28]. Recently, a published report where $600 \mathrm{mg}$ IV every $8 \mathrm{~h}$ was initiated in 5 out of 6 patients with $S$. aureus bacteremia (3 endocarditis, 1 urinary tract infection, 1 ethmoid osteomyelitis with uveitis, and 1 prostatitis with septic thrombophlebitis) demonstrated resolution of infection in 5 out of the 6 patients, including one patient with a vancomycin-intermediate (vancomycin $\mathrm{MIC}=4 \mathrm{mg} / \mathrm{L}$ ) isolate [29]. Recently, Casapao and colleagues [30] described 527 patients who received ceftaroline therapy, including 241 patients infected with MRSA. In this observational study of patients who received ceftaroline for various indications, 76 (14.4\%) received an off-label dose. Side effects were noted to be slightly higher in these patients (17.1\%) but all side effects reported in this study were noted to be $\leq 7.8 \%$ [30]. Although most patients in these reports received multiple agents active against MRSA, the results support further research into the efficacy and safety of ceftaroline at this increased dose. Extended infusion beta-lactam therapy has shown promise in the treatment of ICU infections, notably with piperacillin/tazobactam (given as a 4-h infusion) [31]. Infusion times longer than $2 \mathrm{~h}$ were not feasible in this patient due to a combination of drug stability (manufacturer labeling indicates 6-h stability at room temperature) and shortage of infusion sites from demand for other non-antimicrobial parenteral infusions.

Serum concentrations (obtained to characterize the pathophysiological changes associated with inhalational thermal injuries) demonstrated that serum drug concentrations remained above the MIC ( $0.5 \mathrm{mg} / \mathrm{L}$ by Etest) for the full duration of the dosing interval. The 8-h dosing regimen was continued due to the potential for increased drug clearance noted in 
this patient, as the high inoculum infection, which could impact the efficacy of ceftaroline. This "inoculum effect" has been noted with other beta-lactams, including cephalosporins as well as glycopeptide antimicrobials [32-34]. Our patient demonstrated similar drug clearance and $V_{\mathrm{d}}$ compared to healthy adults. However, this patient also demonstrated a much shorter half-life (1.5 versus $2.66 \mathrm{~h}$ ) and increased AUC (87.6 versus $56.3 \mu \mathrm{g} \mathrm{h} / \mathrm{mL}$ ), when compared with data reported in the ceftaroline prescribing information. This finding was dissimilar to previously published reports characterizing the increased clearance of beta-lactam antimicrobials in patients with thermal injury $[9,11,14]$. This could potentially be due to the limited amount of burn injury $(<1 \%$ total BSA) and time period from the burn injury itself, as the ceftaroline therapy was initiated in this patient many days into their admission. Curiously, the concentration obtained 30-min post-infusion was higher than reported in the prescribing information and cannot be completely explained other than this patient exhibited altered ceftaroline PK distribution. The patient completed a total of 14 days of ceftaroline therapy and responded appropriately with resolution of signs and symptoms of infection, and more importantly with no reported toxicity from antimicrobial therapy. Ultimately, the patient was liberated from mechanical ventilation on HD 22 and discharged to a psychiatric care facility on HD 48.

\section{CONCLUSION}

In conclusion, we describe a case of an inhalational thermal burn injury patient successfully treated with ceftaroline $600 \mathrm{mg}$ IV every $8 \mathrm{~h}$ for documented MRSA pneumonia with a vancomycin MIC of $2 \mathrm{mg} / \mathrm{L}$. We also characterized the PK of ceftaroline in a patient with inhalational thermal injuries utilizing a higher than normal dose of medication with the aim of optimizing serum concentrations due to their disease pathophysiology. Our patient demonstrated higher calculated AUC values resulting from the higher dose and increased frequency of dosing, as well as shorter serum half-life. Larger studies are needed to further evaluate this increased dose for safety and efficacy. Clinicians should recognize these pathophysiological changes in burn injury patients and, if possible, adjust treatment accordingly, thus allowing for improved patient outcomes.

\section{ACKNOWLEDGMENTS}

No funding or sponsorship was received for publication of this work. All named authors meet the International Committee of Medical Journal Editors (ICMJE) criteria for authorship for this manuscript, take responsibility for the integrity of the work as a whole, and have given final approval to the version to be published.

Disclosures. Janie Faris declares she has no conflict of interest. Ryan Mynatt has served on the advisory board for Theravance Pharmaceuticals. Ashley Snyder is currently employed by Merck \& Co, Inc. but was a fellow at the time the work was conducted. Michael J. Rybak has received grants, speaking honoraria or consulting fees for Actavis, Cempra, Cubist (a wholly owned subsidiary of Merck \& Co, Inc.), The Medicines Company, Theravance, and partial funding support from NIH. It is important to note that the treatment described in this case report was prescribed at the treating physician's sole discretion without 
the knowledge of Actavis (formerly Forest Laboratories, Inc.), the marketer of ceftaroline fosamil, or its affiliates. The bioassay results were generated without Actavis's knowledge or authorization. Upon learning of the proposed publication, Actavis was unable to confirm these bioassay results.

Compliance with ethics guidelines. This article does not contain any new studies with human or animal subjects performed by any of the authors. The institutional review board (IRB) of Wayne State University does not require IRB approval for publication of a single case report. This was a retrospective case report, so no consent was required.

Open Access. This article is distributed under the terms of the Creative Commons Attribution-NonCommercial 4.0 International License (http://creativecommons.org/licenses/ by-nc/4.0/), which permits any noncommercial use, distribution, and reproduction in any medium, provided you give appropriate credit to the original author(s) and the source, provide a link to the Creative Commons license, and indicate if changes were made.

\section{REFERENCES}

1. Haque NZ, Zuniga LC, Peyrani P, et al. Relationship of vancomycin minimum inhibitory concentration to mortality in patients with methicillin-resistant Staphylococcus aureus hospital-acquired, ventilator-associated, or health-care-associated pneumonia. Chest. 2010;138(6):1356-62.

2. Jeffres MN, Isakow W, Doherty JA, et al. Predictors of mortality for methicillin-resistant Staphylococcus aureus health-care-associated pneumonia: specific evaluation of vancomycin pharmacokinetic indices. Chest. 2006;130(4):947-55.
3. Sakoulas G, Moise-Broder PA, Schentag J, Forrest A, Moellering RC Jr, Eliopoulos GM. Relationship of MIC and bactericidal activity to efficacy of vancomycin for treatment of methicillin-resistant Staphylococcus aureus bacteremia. J Clin Microbiol. 2004;42(6):2398-402.

4. Ceftaroline fosamil [Prescribing Information]. Forest Pharmaceuticals.2010.

5. Kollef MH. New antimicrobial agents for methicillin-resistant Staphylococcus aureus. Crit Care Resusc. 2009;11(4):282-6.

6. Kosowska-Shick K, McGhee PL, Appelbaum PC. Affinity of ceftaroline and other beta-lactams for penicillin-binding proteins from Staphylococcus aureus and Streptococcus pneumoniae. Antimicrob Agents Chemother. 2010;54(5):1670-7.

7. Steed ME, Rybak MJ. Ceftaroline: a new cephalosporin with activity against resistant gram-positive pathogens. Pharmacotherapy. 2010;30(4):375-89.

8. Steed M, Vidaillac C, Rybak MJ. Evaluation of ceftaroline activity versus daptomycin (DAP) against DAP-nonsusceptible methicillin-resistant Staphylococcus aureus strains in an in vitro pharmacokinetic/pharmacodynamic model. Antimicrob Agents Chemother. 2011;55(7):3522-6.

9. Bonapace CR, White RL, Friedrich LV, Norcross ED, Bosso JA. Pharmacokinetics of cefepime in patients with thermal burn injury. Antimicrob Agents Chemother. 1999;43(12):2848-54.

10. Conil JM, Georges B, Breden A, et al. Increased amikacin dosage requirements in burn patients receiving a once-daily regimen. Int $\mathrm{J}$ Antimicrob Agents. 2006;28(3):226-30.

11. Conil JM, Georges B, Lavit $M$, et al. Pharmacokinetics of ceftazidime and cefepime in burn patients: the importance of age and creatinine clearance. Int $\mathrm{J}$ Clin Pharmacol Ther. 2007;45(10):529-38.

12. Mohr JF 3rd, Ostrosky-Zeichner L, Wainright DJ, Parks DH, Hollenbeck TC, Ericsson CD. Pharmacokinetic evaluation of single-dose intravenous daptomycin in patients with thermal burn injury. Antimicrob Agents Chemother. 2008;52(5):1891-3.

13. Rybak MJ, Albrecht LM, Berman JR, Warbasse LH, Svensson CK. Vancomycin pharmacokinetics in burn patients and intravenous drug abusers. Antimicrob Agents Chemother. 1990;34(5):792-5. 
14. Doh K, Woo H, Hur J, et al. Population pharmacokinetics of meropenem in burn patients. J Antimicrob Chemother. 2010;65(11):2428-35.

15. Gallagher JJ, Williams-Bouyer N, Villarreal C, Heggers JP, Herndon DN. Treatment of infection in burns. In: David NH, editor. Total Burn Care. 3rd ed. Edinburgh: W.B. Saunders; 2007. p. 136-76.

16. Jaehde U, Sorgel F. Clinical pharmacokinetics in patients with burns. Clin Pharmacokinet. 1995;29(1):15-28.

17. Gallagher JJ, Williams-Bouyer N, Villarreal C, Heggers JP, Herndon DN. Treatment of infection in burns. In: Herndon DN, editor. Total burn care. 3rd ed. Philadelphia: Saunders Elsevier; 2007.

18. Traber FL Herdon DN, Enkhbaatar P, Maybauer MO, Maybauer DM. The pathophysiology of inhalation injury. In: Herndon DN, editor. Total burn care. 3rd ed. Philadelphia: Saunders Elsevier; 2007.

19. Lovering AM, Le Floch R, Hovsepian L, et al. Pharmacokinetic evaluation of linezolid in patients with major thermal injuries. J Antimicrob Chemother. 2009;63(3):553-9.

20. Hallam MJ, Allen JM, James SE, et al. Potential subtherapeutic linezolid and meropenem antibiotic concentrations in a patient with severe burns and sepsis. J Burn Care Res. 2010;31(1):207-9.

21. Weinbren MJ. Pharmacokinetics of antibiotics in burn patients. J Antimicrob Chemother. 1999;44(3):319-27.

22. Kulkarni RR, Kulkarni PR. Linezolid-induced near-fatal serotonin syndrome during escitalopram therapy: case report and review of literature. Indian J Psychol Med. 2013;35(4):413-6.

23. Vidaillac C, Leonard SN, Rybak MJ. In vitro activity of ceftaroline against methicillin-resistant Staphylococcus aureus and heterogeneous vancomycin-intermediate $S$. aureus in a hollow fiber model. Antimicrob Agents Chemother. 2009;53(11):4712-7.

24. Corey GR, Wilcox MH, Talbot GH, Thye D, Friedland D, Baculik T. CANVAS 1: the first Phase III, randomized, double-blind study evaluating ceftaroline fosamil for the treatment of patients with complicated skin and skin structure infections. J Antimicrob Chemother. 2010;65(Suppl 4):iv41-51.

25. File TM, Jr., Low DE, Eckburg PB, et al. FOCUS 1: a randomized, double-blinded, multicentre, phase III trial of the efficacy and safety of ceftaroline fosamil versus ceftriaxone in community-acquired pneumonia. J Antimicrob Chemother. 2011;66(Suppl 3):iii19-32.
26. Low DE, File TM, Jr., Eckburg PB, et al. FOCUS 2: a randomized, double-blinded, multicentre, phase III trial of the efficacy and safety of ceftaroline fosamil versus ceftriaxone in community-acquired pneumonia. J Antimicrob Chemother. 2011;66(Suppl 3):iii33-44.

27. Wilcox MH, Corey GR, Talbot GH, Thye D, Friedland D, Baculik T. CANVAS 2: the second Phase III, randomized, double-blind study evaluating ceftaroline fosamil for the treatment of patients with complicated skin and skin structure infections. J Antimicrob Chemother. 2010;65 Suppl 4:iv53-iv65.

28. Bhavnani S, Van Wart S, Rubino C, et al. Pharmacokinetic/pharmacodynamic target attainment analysis to evaluate susceptibility test interpretive criteria for ceftaroline against Staphylococcus aureus. Poster presented at the 51st annual interscience conference on antimicrobial agents and chemotherapy; Chicago, IL, September 17-20, 2011.

29. Ho TT, Cadena J, Childs LM, Gonzalez-Velez M, Lewis JS 2nd. Methicillin-resistant Staphylococcus aureus bacteraemia and endocarditis treated with ceftaroline salvage therapy. J Antimicrob Chemother. 2012;67(5):1267-70.

30. Casapao AM, Davis SL, Barr VO, et al. Large retrospective evaluation of the effectiveness and safety of ceftaroline fosamil therapy. Antimicrob Agents Chemother. 2014;58(5):2541-6.

31. Lodise TP Jr, Lomaestro B, Drusano GL. Piperacillin-tazobactam for Pseudomonas aeruginosa infection: clinical implications of an extended-infusion dosing strategy. Clin Infect Dis. 2007;44(3):357-63.

32. Sabath LD, Garner C, Wilcox C, Finland M. Effect of inoculum and of beta-lactamase on the anti-staphylococcal activity of thirteen penicillins and cephalosporins. Antimicrob Agents Chemother. 1975;8(3):344-9.

33. Nannini EC, Stryjewski ME, Singh KV, et al. Inoculum effect with cefazolin among clinical isolates of methicillin-susceptible Staphylococcus aureus: frequency and possible cause of cefazolin treatment failure. Antimicrob Agents Chemother. 2009;53(8):3437-41.

34. LaPlante KL, Rybak MJ. Impact of high-inoculum Staphylococcus aureus on the activities of nafcillin, vancomycin, linezolid, and daptomycin, alone and in combination with gentamicin, in an in vitro pharmacodynamic model. Antimicrob Agents Chemother. 2004;48(12):4665-72. 